\title{
Glucocorticoids associate with cardiometabolic risk factors in black South Africans
}

\author{
Siphiwe N Dlamini(1,2, Zané Lombard ${ }^{3}$, Lisa K Micklesfield ${ }^{1}$, Nigel Crowther ${ }^{4}$, Shane A Norris ${ }^{1}$, Tracy Snyman ${ }^{4}$, \\ Andrew A Crawford ${ }^{5,6}$, Brian R Walker ${ }^{6,7}$ and Julia H Goedecke ${ }^{101,2}$ \\ ${ }^{1}$ SAMRC/Wits Developmental Pathways for Health Research Unit (DPHRU), School of Clinical Medicine, Faculty of Health Sciences, University of the \\ Witwatersrand, Johannesburg, South Africa \\ ${ }^{2}$ Non-communicable Diseases Research Unit, South African Medical Research Council, Cape Town, South Africa \\ ${ }^{3}$ Division of Human Genetics, National Health Laboratory Service, and School of Pathology, Faculty of Health Sciences, University of the Witwatersrand, \\ Johannesburg, South Africa \\ ${ }^{4}$ Department of Chemical Pathology, National Health Laboratory Service and Faculty of Health Sciences, University of the Witwatersrand, Johannesburg, \\ South Africa \\ ${ }^{5}$ Population Health Sciences, Bristol Medical School, University of Bristol, Bristol, UK \\ ${ }^{6} \mathrm{BHF}$ Centre for Cardiovascular Science, University of Edinburgh, Edinburgh, UK \\ ${ }^{7}$ Institute of Genetic Medicine to Translational \& Clinical Research Institute, Newcastle University, Newcastle upon Tyne, UK
}

Correspondence should be addressed to S N Dlamini: siphiwe208@live.com

\begin{abstract}
Circulating glucocorticoids are associated with metabolic syndrome and related cardiometabolic risk factors in non-Africans. This study investigated these associations in Africans, whose metabolic phenotype reportedly differs from Europeans. Adiposity, blood pressure, glycaemia, insulin resistance, and lipid profile, were measured in 316 African men and 788 African women living in Soweto, Johannesburg. The 2009 harmonized criteria were used to define metabolic syndrome. Serum glucocorticoids were measured using liquid chromatography-mass spectrometry. Cortisol was associated with greater odds presenting with metabolic syndrome (odds ratio $(95 \% \mathrm{Cl})=1.50(1.04,2.17)$ and higher systolic (beta coefficient, $\beta(95 \% \mathrm{Cl})=0.04(0.01,0.08))$ and diastolic $(0.05(0.02,0.09))$ blood pressure, but higher $\operatorname{HDL}(0.10(0.02,0.19))$ and lower LDL $(-0.14(-0.24,-0.03))$ cholesterol concentrations, in the combined sample of men and women. In contrast, corticosterone was only associated with higher insulin sensitivity (Matsuda index; $0.22(0.03,0.41)$ ), but this was not independent of BMI. Sex-specific associations were observed, such that both cortisol and corticosterone were associated with higher fasting glucose (standardized $\beta(95 \% \mathrm{Cl}): 0.24(0.12,0.36)$ for cortisol and $0.12(0.01,0.23)$ for corticosterone $)$ and HbA1c $(0.13(0.01,0.25)$ for cortisol and $0.12(0.01,0.24)$ for corticosterone) in men only, but lower $\operatorname{HbA} 1 \mathrm{c}(0.10(-0.20,-0.01)$ for cortisol and $-0.09(-0.18,-0.03)$ for corticosterone) in women only. Our study reports for the first time that associations between circulating glucocorticoid concentrations and key cardiometabolic risk factors exhibit both glucocorticoid- and sex-specificity in Africans.
\end{abstract}

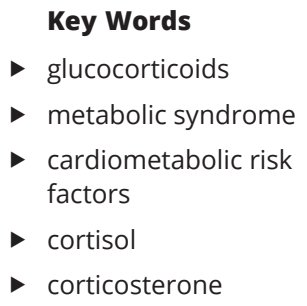

Endocrine Connections (2021) 10, 873-884

\section{Introduction}

Elevated waist circumference, blood pressure, fasting glucose, triglycerides, and reduced high-density lipoprotein (HDL) cholesterol often cluster together as components of metabolic syndrome (1). Chronic exposure to excess cortisol leads to Cushing's syndrome, which exhibits metabolic features similar to metabolic syndrome (2). Accordingly, previous cross-sectional studies suggest that circulating cortisol concentrations are associated with metabolic syndrome, its components, and related cardiometabolic risk factors, including measures of obesity, insulin resistance, and glucose intolerance $(3,4,5,6,7)$.

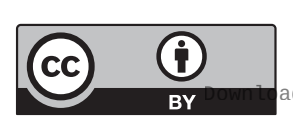

This work is licensed under a Creative Commons Attribution 4.0 International License. 
Cortisol may not be the only glucocorticoid involved in metabolic syndrome as humans also produce corticosterone. As cortisol production is almost ten times higher than corticosterone (8), previous studies have only focused on cortisol and assumed that these two glucocorticoids have identical functions (9). However, recent evidence suggests that cortisol and corticosterone may have distinct roles in human health and disease (10).

Notably, most of the reported cross-sectional studies that have explored the associations between cortisol and cardiometabolic risk factors were conducted in nonAfricans (3, 4, 5, 6). Africans have a different metabolic phenotype to that of their European counterparts $(11,12)$. Some studies have shown that in urban settings Africans have a higher prevalence of obesity and insulin resistance, yet have less visceral adipose tissue (VAT) than Europeans $(13,14,15)$. Although the mechanisms underlying these ethnic differences are not yet known, a recent study suggested that glucocorticoids may be involved (16). Goedecke et al. (16) reported that black South African women demonstrated lower gene expression of the glucocorticoid receptor (GR) in s.c. adipose tissue (SAT) than women of European ancestry. The lower GR expression in these African women was associated with less VAT, higher SAT inflammation, and whole-body lower insulin sensitivity. Furthermore, some studies have shown that circulating cortisol concentrations are lower in African women compared to women of European ancestry $(17,18)$. In support of these ethnic differences, a study that included both African men and women suggested more evidence of hypothalamic-pituitary-adrenal (HPA) axis dysregulation in African males compared to their European ancestry counterparts, and the HPA axis dysregulation was associated with an increased risk of cardiovascular disease (7). Based on the above observations, we hypothesized that the often-observed ethnic differences in body fat distribution, inflammatory profile, insulin sensitivity, and circulating cortisol concentrations are partly explained by tissue-specific differences of glucocorticoid metabolism within SAT.

However, as studies of cortisol and metabolic syndrome in Africans are scarce and none have included corticosterone, it is still uncertain whether circulating glucocorticoids associate with metabolic syndrome and related cardiometabolic risk factors in Africans (19, 20, 21). Therefore, the aim of this study was to investigate the associations between fasting serum corticosterone and cortisol concentrations and metabolic syndrome and related cardiometabolic risk factors in black South African men and women.

\section{Materials and methods}

\section{Study population and sampling procedure}

The study sample consisted of adult male and female participants recruited from various studies at the SAMRC/ Wits Developmental Pathways for Health Research Unit, based at the Chris Hani Baragwanath Academic Hospital in Soweto, Johannesburg. Study sample 1 (ethical clearance M090620) included women recruited as part of the Africa Wits-INDEPTH partnership for Genomic Research (AWI-Gen) study (22). These women $(n=1007)$ who were randomly recruited between 2011 and 2013 were the caregivers of the birth to twenty plus (Bt20+) cohort. Bt20+ is an ongoing longitudinal study, and its profile has been described elsewhere (23). The majority of the caregivers of the Bt20+ participants are South African men and women of African ancestry who were self-identified as South-Eastern Bantu language speakers, and only this ethnic group was included in the present study. Details of data collection protocols for the AWI-Gen study have been described elsewhere (24). Study sample 2 included participants (501 men and 529 women) recruited as part of a longitudinal study designed to identify the determinants of type 2 diabetes risk in middle-aged South African men and women (ethical clearance M160604). These participants were a subsample of the AWI-Gen study from whom follow-up data collected between January 2017 and August 2018 were used and included no participants from study sample 1.

The selection of the participants for the present study was primarily based on the availability of serum samples for glucocorticoid determination. Participants who were pregnant or using corticosteroid medication were excluded from the study (Fig. 1). A total of 1104 participants were selected for the present study, with the selection process described in Fig. 1. Consent was obtained from all study participants following a full explanation of the purpose and nature of all procedures used. Further ethical clearance for all analyses conducted as part of the present study was also obtained (ethical clearance no: M160225) from the University of the Witwatersrand Human Research Ethics Committee (Medical).

\section{Participant testing procedures}

Participants were asked to remove heavy clothes and shoes prior to weight and height measurements. Weight was measured using a calibrated digital scale (Dismed, Halfway House, SA) to the nearest $0.1 \mathrm{~kg}$. Standing height was measured using a fixed-wall stadiometer (Holtain, Crymych, UK) to the nearest $0.1 \mathrm{~cm}$. BMI was calculated

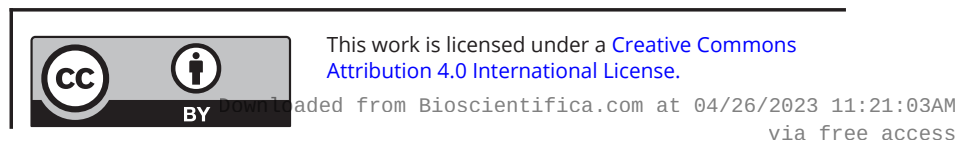




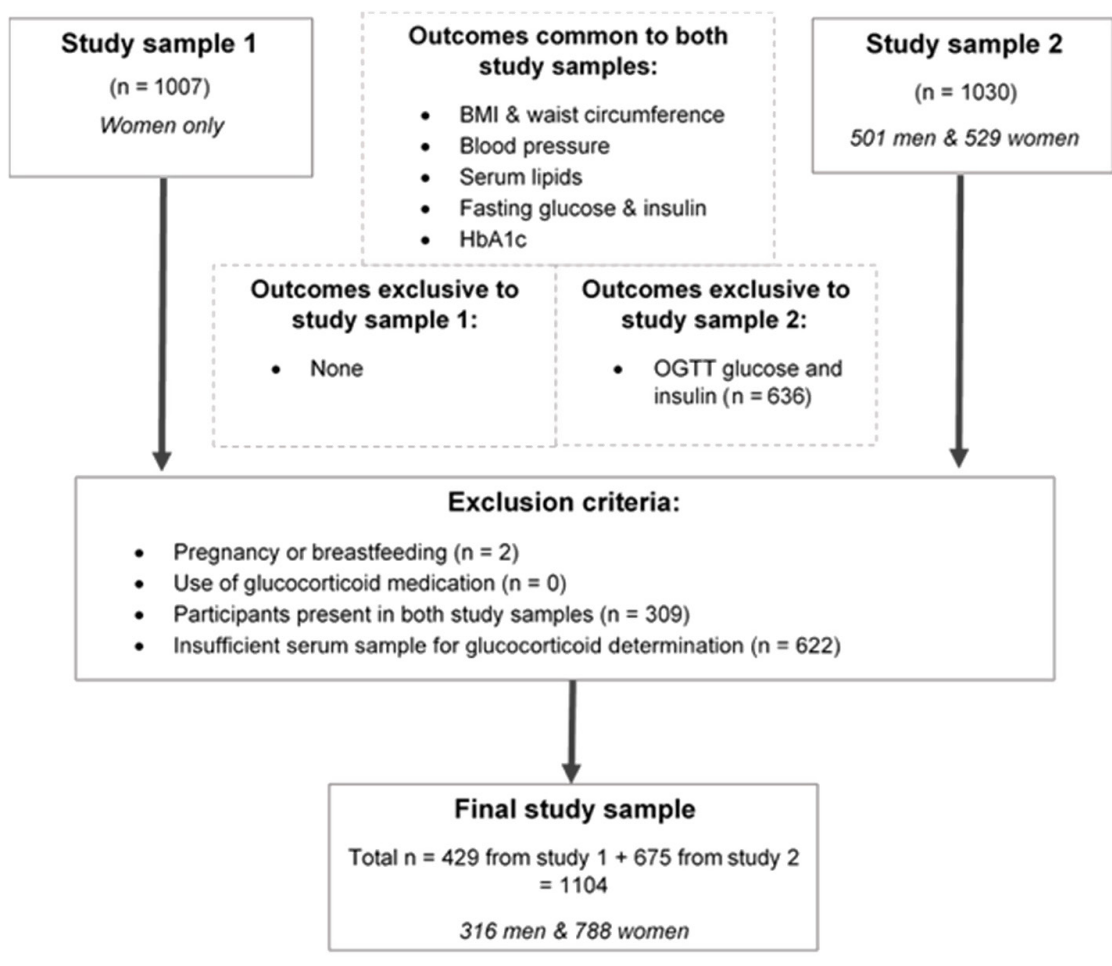

Figure 1

Selection of the study sample. as weight (kilograms) divided by height squared (metre square). Waist circumference was determined at the level of the umbilicus when the participants were standing and measured using a soft measuring tape to the nearest $0.5 \mathrm{~cm}$. Systolic and diastolic blood pressure were measured on the left arm using a digital blood pressure reader (Omron M6, Kyoto, Japan) and appropriate cuffs. After the participant had been seated for at least $5 \mathrm{~min}$, three blood pressure readings were taken at 2 -min intervals, and the average of the second and third readings was recorded and used in the analyses. Participants who self-reported as smoking any form of tobacco and/or drinking any form of alcohol were classified as current smokers or drinkers, respectively. Participants were asked to bring their chronic medication to the interview sessions to confirm their disease status.

\section{Blood sampling and biochemical analyses}

Standard venepuncture techniques were used to collect blood samples after 10-12 h of fasting for the subsequent determination of serum lipids, HbA1c, serum insulin, plasma glucose, and serum glucocorticoid concentrations. Blood sampling time for the fasting samples was recorded and ranged from 07:00 to 11:59 h. To convert the blood sampling time into a more continuous variable, minutes from the earliest blood sample (07:00 h) were calculated. For example, a blood sample collected at 08:00 h was assigned a time value of $60 \mathrm{~min}$. A standard oral glucose tolerance test (OGTT) was conducted on a sub-sample of participants (Study 2 in Fig. 1, $n=636$ ). During the OGTT, the participants ingested $75 \mathrm{~g}$ anhydrous glucose dissolved in $250 \mathrm{~mL}$ water. Blood samples were then collected at 30-min intervals for $120 \mathrm{~min}$ for the subsequent determination of the Matsuda index (OGTT-derived insulin sensitivity) (http://mmatsuda.diabetes-smc.jp/english.html) and 2-h OGTT glucose concentrations.

Serum lipid concentrations, including triglycerides and total- and HDL-cholesterol, were measured using prescribed enzymatic methods with the RX Daytona Chemistry Analyser (Randox, Crumlim, UK). The interand intra-assay coefficients of variation (CVs) for the serum lipids were 1.4 and $1.5 \%$ for triglycerides, respectively, both $1.3 \%$ for total cholesterol and 1.7 and 1.8\% for HDL cholesterol, respectively. Serum low-density lipoprotein (LDL)-cholesterol concentrations were calculated using the Friedewald equation (25). The HbA1c concentrations were measured in whole blood samples using the D-10 haemoglobin testing system (BioRad), and the interand intra-assay CVs were both $1.8 \%$. Plasma glucose concentrations were measured using the RX Daytona Chemistry Analyser (Randox), and the Advia 1800 Chemistry Analyser (Siemens Healthcare Diagnostics) was used to measure serum insulin concentrations. The inter- and intra-assay CVs were 3.3 and $1.3 \%$ for glucose, respectively and 2.9 and $2.7 \%$ for insulin, respectively. The HOMA2-IR calculator version 2.2.3 was used to estimate

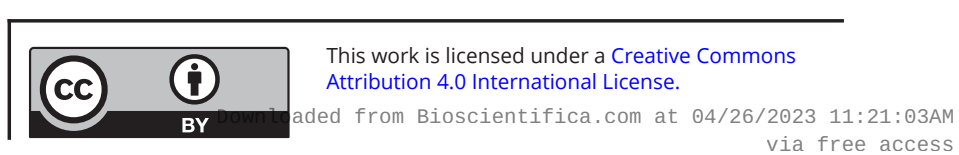


insulin resistance levels (https://www.dtu.ox.ac.uk/ homacalculator) from fasting serum insulin and plasma glucose concentrations.

\section{Definition of metabolic syndrome}

The 2009 harmonized guidelines were used for the diagnosis of metabolic syndrome (1). The harmonized diagnosis requires the presence of three or more of the following criteria: elevated waist circumference $(\geq 94 \mathrm{~cm}$ in men, $\geq 80 \mathrm{~cm}$ in women), elevated triglyceride $(\geq 1.7 \mathrm{mmol} / \mathrm{L})$, low HDL cholesterol ( $<1.0 \mathrm{mmol} / \mathrm{L}$ in males, $<1.3 \mathrm{mmol} / \mathrm{L}$ in females), elevated blood pressure (systolic $\geq 130$ and/or diastolic $\geq 85 \mathrm{mmHg})$, and elevated fasting glucose $(\geq 5.6$ $\mathrm{mmol} / \mathrm{L})$. The use of prescribed medications for elevated blood pressure and diabetes were also considered as relevant indicators of the presence of elevated blood pressure and fasting blood glucose concentrations, respectively.

\section{Steroid extraction and glucocorticoid measurements}

\section{Extraction of steroids from serum}

All calibrators, internal standards (labelled with stable isotope deuterium; corticoseterone-d8 and cortisol-d4), and controls were purchased from Chromsystems (Grafelfing, Germany). A liquid-liquid extraction technique (Agilent technologies) was used to extract all steroids. Briefly, $50 \mu \mathrm{L}$ of the internal standard was added to $500 \mu \mathrm{L}$ of serum. Three millilitres of methyl tert-butyl ether (MTBE) were added to each sample and vortexed at maximum speed of $3 \times 30 \mathrm{~s}$, with $30 \mathrm{~s}$ rests in between. The sample was then centrifuged at $1372 \boldsymbol{g}$ for $10 \mathrm{~min}$. The supernatant was transferred into a clean glass tube and dried down using technical grade nitrogen (Afrox, Johannesburg, SA) at room temperature. The extracted steroids were reconstituted in $100 \mu \mathrm{L}$ of $50 \%$ methanol: water and then transferred into limited volume analytical vials (Kinesis inc., Vernon Hills, USA) for analyses on the ultra-higher pressure liquid chromatography (UPLC) mass spectrometry (MS).

\section{Glucocorticoid measurement in the UPLC-MS}

Ten microlitres of each extracted sample were injected onto a Kinetex F5 $2.6 \mu \mathrm{m} 50 \times 2.1 \mathrm{~mm}$ column (Phenomenex, California, USA) utilizing an Acquity UPLC that was connected to a Xevo-TQS MS (Waters Corporation, Milford, USA). The UPLC-MS method was optimized and validated to quantitate both corticosterone and cortisol. A flow rate of $0.6 \mathrm{~mL} / \mathrm{min}$ set on a gradient profile was used to elute the compounds. A binary flow mobile phase was used and consisted of mobile phase A: $5 \mathrm{mM}$ ammonium formate and $0.1 \%$ formic acid in deionized water and mobile phase B: $1 \mathrm{mM}$ ammonium formate and $0.1 \%$ formic acid in acetonitrile to elute off each of the glucocorticoids over a total run time of $5.50 \mathrm{~min}$. The gradient programme was set as follows: 5\% B for the first $0.95 \mathrm{~min}$; 30\% B for $0.5 \mathrm{~min}$; 70\% B for $0.50 \mathrm{~min}$; this was ramped to $90 \% \mathrm{~B}$ at $1.95 \mathrm{~min}$ and maintained for $2.55 \mathrm{~min}$ with a final ramp to 95\% B for 0.50 min to wash the column. The conditions were then changed back to initial starting conditions $(5 \%$ B) at $4 \mathrm{~min}$ to equilibrate the column. The retention time for corticosterone and corticosterone-d8 was 3.23 and 3.25 min, respectively, whereas the retention time for cortisol and cortisol-d4 was 3.35 and $3.36 \mathrm{~min}$, respectively.

Electrospray ionization in positive mode was used in the MS. Capillary voltage was $3 \mathrm{kV}$ with cone and collision energies optimized to each compound analysed. Source temperature and desolvation temperature were set at $150^{\circ}$ and $350^{\circ} \mathrm{C}$, respectively. Cone gas was set at $550 \mathrm{~L} / \mathrm{h}$, and desolvation gas was set at $150 \mathrm{~L} / \mathrm{h}$. The analytes were identified with multiple reaction monitoring identified for each individual compound. Corticosterone had a transition of $347>121$ as the quanitifier and $347>329$ for the qualifier. Cortisol utilized the transition of $363>121$ for the quantifier and 363>97 for the qualifier.

The inter- and intra-assay CVs for serum corticosterone were 7.3 and 2.9\%, respectively, and for cortisol concentrations were 13.6 and $9.6 \%$, respectively on control level 1 (lowest concentration).

\section{Statistical analyses}

All statistical analyses were conducted in $\mathrm{R}$ version 3.6.2 (26). Prior to the statistical analyses, the normality of the continuous variables was assessed using distribution graphs (QQ-plots, histograms, and box plots) as well as the Shapiro-Wilk test. None of the continuous variables shown in Table 1 were normally distributed and are thus presented as medians (25th-75th percentiles). Therefore, the Mann-Whitney $U$-test was used to assess the statistical differences in the continuous variables between men and women (Table 1). Differences in the categorical variables (prescribed medication, prevalence of metabolic syndrome, and lifestyle factors) are presented as observations/total non-missing observations $(n / N(\%))$ and were compared between men and women using a chi-square test (Table 1).

Logistic or linear regression models were used to determine associations between serum glucocorticoid

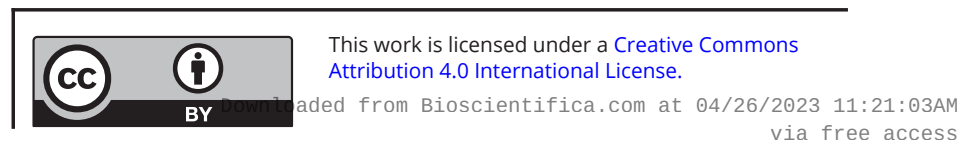


The tested outcome variables included metabolic syndrome, BMI, waist circumference, systolic and diastolic blood pressure, fasting glucose and insulin, 2-h glucose, HOMA2-IR, Matsuda index, and serum lipids (triglyceride, total-, LDL- and HDL-cholesterol).

Data from the logistic regression models are presented as the odds ratio presenting with metabolic syndrome, for both corticosterone and cortisol, with adjustment for potential confounders (age, sex, blood sampling time, smoking, and alcohol) (Fig. 2). For the linear models, the beta coefficients and 95\% CI for the various outcome measures listed above are presented in Table 2 adjusted for the potential confounders only (referred as Model 1 ) and for the potential confounders plus BMI (referred as Model 2).

\section{Results}

\section{Participant characteristics}

The characteristics of the whole sample, and men and women separately, are presented in Table 1, with a comparison of the categorized components of metabolic syndrome and related cardiometabolic risk factors presented in Supplementary Table 1 (see section on supplementary materials given at the end of this article). Women were younger than men $(P<0.001)$ and had a higher BMI $(P<0.001)$ and waist circumference $(P=0.029)$ (Table 1$)$. Accordingly, a greater proportion of women was classified with obesity $(65.2 \%$ vs $25.3 \% ; P<0.001$ ) and with a waist circumference greater than that of metabolic syndrome cut points $(88.3 \%$ vs 48.4\%; $P<0.001$ ) (Supplementary Table 1).

Despite differences in obesity, fasting glucose was similar between men and women. However, compared

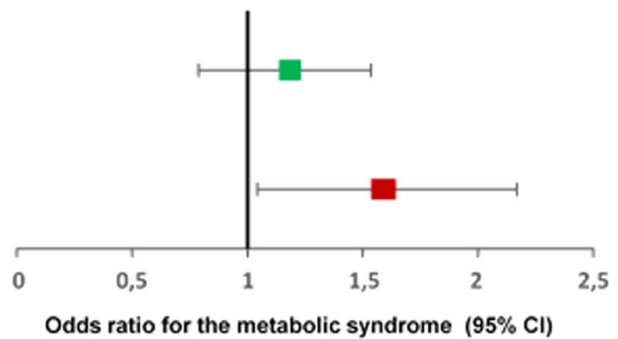

\begin{tabular}{lrclr} 
& Odds ratio & $95 \% \mathrm{Cl}$ & $\mathbf{p}$ & \multicolumn{1}{c}{ Sex Int } \\
Corticosterone & 1.104 & $(0.792,1.540)$ & 0.558 & 0.142 \\
Cortisol & 1.502 & $(1.044,2.170)$ & 0.029 & 0.245
\end{tabular}

Figure 2

Associations between glucocorticoids and metabolic syndrome. $P, P$ value for the logistic regression model; Sex Int, $P$ value for sex interaction Models adjusted for age, blood sampling time, sex, smoking, and alcohol. to men, women had higher fasting insulin, HOMA2-IR, 2-h glucose, HbA1c concentrations, and lower insulin sensitivity (Matsuda index) (all $P<0.001$ ). Although a larger proportion of women was using diabetes medication (11.2\% vs $6.1 \% ; P=0.022$ ) than men (Table 1 ), when using metabolic syndrome criteria (fasting blood glucose $\geq 5.6$ $\mathrm{mmol} / \mathrm{L}$ and/or using diabetes medication) the proportion of men and women with elevated blood glucose did not differ (Supplementary Table 1).

Although HDL-cholesterol and triglycerides did not differ by sex, total cholesterol and LDL-cholesterol concentrations were higher in women compared to men $(P<0.001$ for both) (Table 1). Moreover, a greater proportion of women had low HDL cholesterol (57.0\% vs $18.0 \%)$, but the prevalence of elevated triglycerides was greater in men $(15.2$ vs $8.2 \%)(P<0.001$ for both) (Supplementary Table 1). There were no observed differences in the use of cholesterol-lowering medication between the sexes.

Systolic and diastolic blood pressure were both higher in men ( $P=0.017$ and $P=0.003$, respectively) (Table 1$)$, and this finding was accompanied by a greater proportion of men having elevated systolic $(\geq 130 \mathrm{mmHg}$ and/or using blood pressure medication, $53.0 \%$ vs $46.3 \%$; $P=0.017$ ), diastolic $(\geq 85 \mathrm{mmHg}$ and/or using blood pressure medication, 61.3 vs $52.8 \%$; $P=0.003)$, and overall blood pressure $(73.4 \%$ vs $66.5 \% ; P=0.030$ ) (Supplementary Table 1). In contrast, a greater proportion of women used blood pressure medication ( $37.9 \%$ vs $27.1 \% ; P=0.002)$.

A greater proportion of women than men were classified as having metabolic syndrome ( $47.6 \%$ vs $26.3 \%$; $P<0.001)$. Meanwhile, a greater proportion of men smoked $(50.8 \%$ vs $8.3 \% ; P<0.001)$ and drank alcohol $(71.7 \%$ vs $28.7 \% ; P<0.001)$ compared to women.

When comparing the serum glucocorticoid concentrations (Table 1), women had higher serum concentrations of both corticosterone $(P<0.001)$ and cortisol $(P<0.002)$. These differences were accompanied by later average blood sampling times (measured as minutes from the earliest blood sampling time) in women compared to men $(P<0.001)$. Notably, the sex differences in serum glucocorticoid concentrations were observed even after adjusting for sampling time, measures of adiposity (BMI and waist circumference), and/or other potential confounders (age, smoking, alcohol) (results not shown).

\section{Associations with metabolic syndrome and related cardiometabolic risk factors in black South Africans}

Only regression models that either showed evidence of associations $(P<0.05)$ or sex-interaction (Sex Int: $P<0.05)$

This work is licensed under a Creative Commons Attribution 4.0 International License. ded from Bioscientifica.com at 04/26/2023 11:21:03AM 
Table 2 Associations of glucocorticoids with cardiometabolic risk factors in African men and women.

\begin{tabular}{l}
\hline \\
\hline BMI \\
Corticosterone \\
Cortisol \\
Systolic BP \\
Corticosterone \\
Cortisol \\
Diastolic BP \\
Corticosterone \\
Cortisol \\
Fasting glucose \\
Corticosterone \\
Cortisol \\
2-h glucose \\
Corticosterone \\
Cortisol \\
HbA1c \\
Corticosterone \\
Cortisol \\
Matsuda index \\
Corticosterone \\
Cortisol \\
LDL cholesterol \\
Corticosterone \\
Cortisol \\
HDL cholesterol \\
Corticosterone \\
Cortisol \\
\hline Ba
\end{tabular}

\begin{tabular}{c}
\hline Model 1 (adjusted \\
\hline Beta $(95 \% \mathrm{Cl})$ \\
\hline$-0.046(-0.096,0.004)$ \\
$-0.011(-0.064,0.041)$ \\
$0.007(-0.029,0.043)$ \\
$0.042(0.005,0.079)$ \\
$0.009(-0.023,0.041)$ \\
$0.051(0.017,0.085)$ \\
$-0.008(-0.055,0.039)$ \\
$0.042(-0.010,0.094)$ \\
$-0.017(-0.099,0.065)$ \\
$-0.013(-0.099,0.074)$ \\
$-0.013(-0.045,0.019)$ \\
$-0.001(-0.036,0.035)$ \\
$0.220(0.034,0.407)$ \\
$0.083(-0.117,0.284)$ \\
$-0.095(-0.193,0.003)$ \\
$-0.138(-0.241,-0.034)$ \\
$0.018(-0.066,0.102)$ \\
$0.105(0.017,0.193)$ \\
\end{tabular}

\begin{tabular}{|c|c|c|}
\hline \multicolumn{3}{|c|}{ Model 2 (adjusted for confounders and BMI) } \\
\hline Beta $(95 \% \mathrm{Cl})$ & $P$ & Sex Int \\
\hline $0.014(-0.021,0.049)$ & 0.430 & 0.956 \\
\hline $0.043(0.007,0.080)$ & 0.018 & 0.634 \\
\hline $0.016(-0.016,0.047)$ & 0.324 & 0.775 \\
\hline $0.052(0.019,0.085)$ & 0.002 & 0.546 \\
\hline$-0.001(-0.048,0.046)$ & 0.953 & 0.008 \\
\hline $0.044(-0.008,0.095)$ & 0.097 & $<0.001$ \\
\hline$-0.001(-0.082,0.079)$ & 0.972 & 0.824 \\
\hline$-0.010(-0.095,0.075)$ & 0.810 & 0.047 \\
\hline$-0.010(-0.042,0.022)$ & 0.543 & 0.002 \\
\hline$-0.001(-0.036,0.035)$ & 0.974 & 0.001 \\
\hline $0.134(-0.036,0.303)$ & 0.122 & 0.921 \\
\hline $0.048(-0.133,0.229)$ & 0.603 & 0.848 \\
\hline$-0.086(-0.184,0.012)$ & 0.085 & 0.029 \\
\hline$-0.136(-0.239,-0.034)$ & 0.009 & 0.768 \\
\hline$-0.009(-0.088,0.071)$ & 0.830 & 0.377 \\
\hline $0.102(0.019,0.186)$ & 0.017 & 0.083 \\
\hline
\end{tabular}

Beta, beta coefficient; Confounders, age, blood sampling time, sex, smoking, alcohol, and relevant medication; $P$ :, $P$ value for the linear regression model; Sex Int, $P$ value for sex interaction. Bold indicates $P<0.05$.

are presented in Fig. 2 and Table 2. The unadjusted models and all other models that were tested are presented in Supplementary Table 2. Figure 2 shows that serum cortisol but not corticosterone concentrations were associated with greater odds presenting with metabolic syndrome. The association between circulating glucocorticoids and metabolic syndrome did not differ by sex (Sex Int: $P=0.142$ for corticosterone and $P=0.245$ for cortisol).

There was no evidence of an association between corticosterone and cortisol and measures of adiposity (BMI and waist circumference) (Table 2). However, there was a sex-interaction for the association between serum cortisol concentration and BMI (Table 2), but the sex-stratified analyses revealed no sufficient evidence of association (Fig. 3). There was no evidence of sex interaction in the associations between either of the serum glucocorticoid measures and waist circumference.

Serum cortisol, but not corticosterone, concentrations were associated with higher systolic $(P=0.028)$ and diastolic $(P=0.004)$ blood pressure, even after adjusting for BMI ( $P=0.018$ and $P=0.002$, respectively). These associations did not differ by sex (Table 2 ).
No evidence of association for serum corticosterone and cortisol concentrations was observed with measures of glycaemia in the combined sample of men and women. However, there was evidence of sex interactions for associations with fasting blood glucose (Sex Int: $P=0.009$ and $P<0.001$, respectively) and HbA1c (Sex Int: $P=0.003$ for both) concentrations (Fig. 3 and Table 2). These sex interactions were present even after adjusting for BMI. Subsequent sex-stratified analyses revealed that both serum corticosterone and cortisol concentrations were associated with higher fasting blood glucose $(P=0.040$ and $P<0.001$, respectively) and HbA1c $(P=0.035$ and $P=0.030$, respectively) concentrations in men only, but lower HbA1c concentrations ( $P=0.042$ and $P=0.030$, respectively) in women only (Fig. 3). Although the association between serum cortisol and 2-h OGTT glucose concentrations showed a sex-interaction after adjusting for BMI (Sex Int: $P=0.047$ ), sex-stratified analyses revealed no evidence of association in either sex (Fig. 3 and Table 2).

Serum corticosterone, but not cortisol, was associated with higher insulin sensitivity (Matsuda index, $P=0.021$ ), but the association was no longer present after adjusting

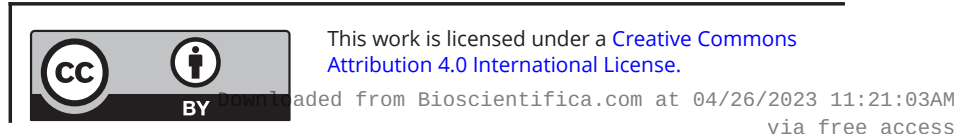



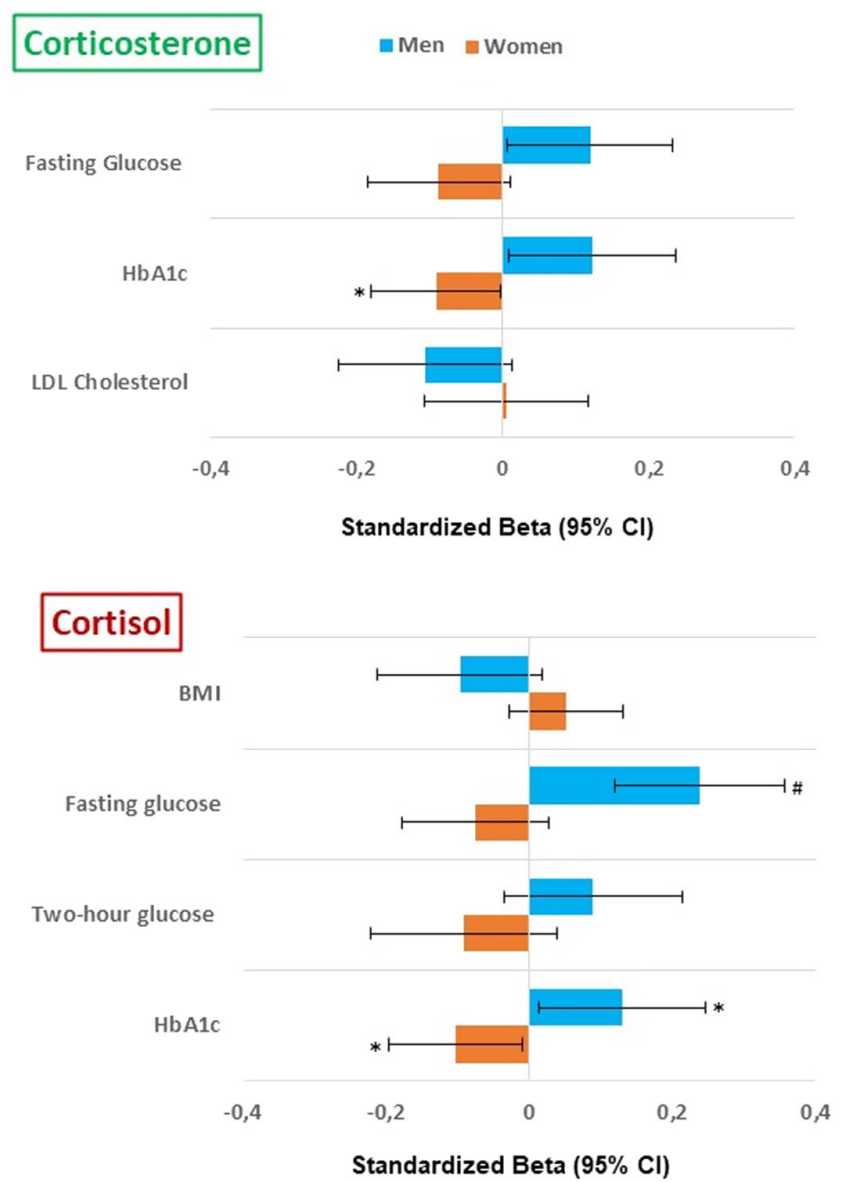

Figure 3

Sex-stratified associations of corticosterone and cortisol with cardiometabolic risk factors. Models adjusted for age, blood sampling time, smoking, alcohol, relevant medication, and $\mathrm{BMI} * P$ value $<0.05$; $\# P$ value $<0.001$

for BMI $(P=0.122)$ (Table 2$)$. Neither of the glucocorticoids were associated with fasting insulin concentrations or HOMA-IR. Moreover, there was no evidence of sex interactions for any measure of insulin resistance.

Serum cortisol concentrations were associated with lower LDL $(P=0.009)$ and higher HDL $(P=0.020)$ cholesterol (Table 2). These associations remained even after adjusting for BMI ( $P=0.009$ and 0.017 , respectively). While serum corticosterone concentrations were not associated with any of the serum lipid concentrations, there was evidence of sex-interaction for LDL cholesterol (Sex Int: $P=0.019$ ) which was not altered by the inclusion of BMI (Sex Int: $P=0.029$ ) (Table 2). However, sex-stratified analyses revealed no evidence of sex-specific associations for LDL cholesterol concentrations (Fig. 3). In contrast, although there was evidence of a sex interaction for HDL cholesterol concentrations (Sex Int: $P=0.038$ ), this was not observed after including BMI as a covariate.

\section{Discussion}

We have shown for the first time that associations between circulating glucocorticoid concentrations and key cardiometabolic variables are both glucocorticoid- and sex-specific in black South Africans.

The higher prevalence of metabolic syndrome in women compared to men in the present study $(45.8 \%$ vs $27.8 \%$ ) was in accordance with previous studies in African populations (28). However, this is the first study to show that fasting serum cortisol concentrations were associated with greater odds of having metabolic syndrome in a combined sample of African men and women. This observation is in accordance with cross-sectional studies from Asian and European populations, which demonstrated that individuals with metabolic syndrome had higher circulating cortisol concentrations compared to those without metabolic syndrome $(6,29)$. The relationship between circulating cortisol concentrations and metabolic syndrome may have been attributed to underlying associations of cortisol with the individual components of metabolic syndrome. In this study, the relationship between cortisol and metabolic syndrome was largely driven by blood pressure as there was no evidence of association with waist circumference or triglycerides, while the association was positive with HDL cholesterol and showed sex-specificity for fasting glucose.

The finding that cortisol, but not corticosterone, concentrations were associated with higher systolic and diastolic blood pressure was in accordance with previous studies demonstrating that circulating cortisol concentrations were associated with both systolic and diastolic blood pressure in Africans, Asians, and Europeans $(19,20,21,30,31)$. A study that has investigated circulating corticosterone in Europeans demonstrated that the associations between corticosterone concentrations and systolic blood pressure were weaker than those demonstrated for cortisol, and corticosterone was not associated with systolic blood pressure in other independent European cohorts (Mackenzie SD, Crawford AA, Ackermann D, Schraut KE, Hayward C, Bolton JL, Saunders C, Al-Dujaili E, Dick B, Escher G, Vogt B, Pruijm M, Ponte B, Wilson JF, Strachan MWJ, Price JF, Phillips DIW, MacKenzie SM, Davies E, Reynolds RM \& Walker BR, unpublished observations). Glucocorticoids influence blood pressure via several mechanisms which are thought to occur through binding of the GR or mineralocorticoid receptor (MR) in the kidneys or vascular system (32). For example, in the kidneys, glucocorticoids impair nitric oxide-mediated renal vasodilation (33) possibly

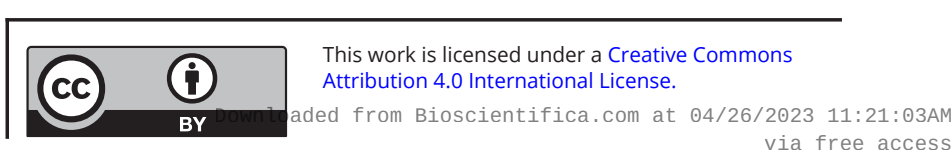


by suppressing nitric oxide synthase, but also influence salt and water retention, ultimately increasing blood pressure (34). There is, however, no strong evidence to suggest that corticosterone and cortisol bind differently to the GR or MR and that these mechanisms are directly involved in the different associations of the two glucocorticoids with blood pressure $(35,36)$. It is unlikely that the glucocorticoid-specific associations with blood pressure were due to lower circulating corticosterone concentrations compared to cortisol (6.3 vs 182.5 $\mathrm{nmol} / \mathrm{L}$ ), as there was evidence of an association between corticosterone and measures of insulin sensitivity, in the present study. While future studies are still required to confirm that corticosterone induces the GR or MR response at these low concentrations in humans, animal studies have suggested that these glucocorticoid-specific associations may be related to differential transmembrane transport by $\mathrm{ABC}$ transporters $(10,37,38)$. This hypothesis originates from the fact that cortisol and corticosterone differ in their susceptibility to transmembrane transport by $\mathrm{ABC}$ transporters. For example, $\mathrm{ABCB} 1$ is an $\mathrm{ABC}$ transporter that is highly expressed in the CNS, and selectively exports cortisol but not corticosterone across the blood-brain barrier (38). Consequently, while corticosterone concentrations are approximately $5 \%$ of the concentration of cortisol in the blood, the concentrations are much higher ( 30\%) in the cerebrospinal fluid (39). Thus, corticosterone may contribute disproportionately to the negative feedback control of the HPA axis within the CNS. As both glucocorticoids similarly bind to the GR and MR to increase blood pressure (33), higher circulating concentrations of corticosterone are accompanied by a more robust HPA axis regulatory response, in comparison to cortisol, to inhibit overall excess glucocorticoid production, ultimately lowering insulin resistance and blood pressure (38).

Additionally, it has been suggested that the relationship between glucocorticoids and elevated blood pressure may be partly mediated by adiposity (40), which is often associated with the other related cardiometabolic risk factors (1). However, adjusting for either BMI or waist circumference did not alter the relationship with blood pressure in our study. Further, there was no evidence of associations between the glucocorticoids and measures of adiposity in this study. It is possible that the overall effects on BMI and waist circumference were confounded by sex differences in body fat distribution and consequent differences in glucocorticoid clearance, as men generally accumulate more VAT compared to women, and glucocorticoid clearance is higher in VAT compared to SAT (41). In this study, men had lower a prevalence of obesity and elevated waist circumference compared to women (25.3 vs $65.2 \%$ and 48.4 vs $88.3 \%$, respectively), and there was evidence of sex interaction in the association between cortisol and BMI ( $P$ value for sex interaction $=0.040$ ). Although sex-stratified analyses revealed that the association between cortisol and BMI was negative in men but positive in women, there was not sufficient evidence $(P>0.05)$ for these relationships in our study sample. Hence, further studies that include larger samples of men and women are required to explore these sex-specific interactions.

Although there was no association between cortisol and insulin resistance, we have shown for the first time that corticosterone was associated with higher insulin sensitivity, as assessed using the Matsuda index. However, this relationship was not present after adjusting for BMI. This finding highlights the importance of including BMI as an additional covariate in glucocorticoid studies and supports the notion that circulating glucocorticoids are associated with adiposity but the relationship is confounded by other factors such as increased metabolic clearance in obesity and type 2 diabetes (27).

This study has also demonstrated that cortisol was associated with a favourable lipid profile (lower serum LDL and higher HDL cholesterol) in black South Africans. Studies from non-Africans that investigated the relationship between circulating cortisol and serum lipid concentrations are inconsistent $(29,30,31,42,43)$. Although some studies reported no association between circulating cortisol and HDL cholesterol $(29,30)$, other studies reported an inverse association (31), whilst another reported a positive association (42). Likewise, while some studies have demonstrated a positive association between circulating cortisol and LDL cholesterol (43), other studies showed no evidence of this association (29, 30). These contradictory findings were thought to be related to differences in age and BMI between the various studies. However, in the present study, the associations between serum cortisol and HDL and LDL cholesterol were independent of BMI, negating the involvement of adiposity-related mechanisms. Indeed, further studies are necessary to understand the exact mechanisms involved.

In addition to glucocorticoid-specific associations, findings from the present study suggested for the first time that both serum corticosterone and cortisol showed sex-specific associations with measures of glycaemia. Both fasting serum cortisol and corticosterone concentrations were associated with higher fasting plasma glucose and HbA1c concentrations in men only, but lower HbA1c

This work is licensed under a Creative Commons Attribution 4.0 International License. ed from Bioscientifica.com at 04/26/2023 11:21:03AM 
concentrations in women only. As these sex-specific associations were similar for both corticosterone and cortisol, mechanisms may involve sex differences in glucocorticoid signalling in the liver, as demonstrated in rodent models. Mice treated with dexamethasone (a synthetic glucocorticoid) demonstrated several sexspecific transcriptional responses in the hepatic tissue $(44,45)$. Moreover, another study showed that chronic exposure to excess glucocorticoid concentrations increased circulating glucose concentrations in male mice, but female mice were protected from these effects (46). Both corticosterone and cortisol are known to increase circulating glucose concentrations by promoting gluconeogenesis in the liver (47). Hence, sex disparities in glucocorticoid signalling within the liver may partly explain the observed sex-specific associations between circulating glucocorticoids and measures of glycaemia, which in turn may be mediated by sex hormones and body fat distribution (48). Indeed, disparities in sex hormone and body fat distribution profiles are profound between men and women. In men, testosterone predominates, and fat is preferentially stored as VAT, whilst in women, oestrogen predominates, and fat is preferentially stored as SAT (45). Such differences may have explained the observed sex-specific associations in the present study. Both sex hormones and fat distribution have been shown to potentially modulate responses to glucocorticoids (48). For example, oestrogen increases corticosteroid-binding globulin, the primary glucocorticoid-binding protein in circulation, consequently increasing biologically active glucocorticoids (49). Likewise, the GR is more highly expressed in VAT than SAT (50). Therefore, further studies are required to investigate the role of hepatic glucocorticoid signalling and interactions with sex hormones and body fat distribution, in the observed sexspecific associations.

This study has some limitations. The role of body distribution, menopausal status, and oral contraceptive use was not assessed in the present study. These factors are known to influence circulating glucocorticoid concentrations and may have influenced some of the reported results $(41,51)$. Women were overrepresented in the present study, and this is common in many epidemiological studies in Africans (52). Although associations with several outcome variables were tested, adjustment for multiple testing was not conducted. However, as the outcomes are all thought to be interrelated cardiometabolic risk factors, adjusting for multiple testing may be too conservative and limit the probability of showing true effects. Additionally, circulating glucocorticoids are often measured at a set time (e.g. 08:00 or 09:00 h) due to their diurnal nature. Notably, blood sample times in the present study varied widely. However, all samples were taken in the morning and blood sampling time was adjusted for in all models.

Regardless of these limitations, this is the largest study to date that has compared the associations of both corticosterone and cortisol concentrations with metabolic syndrome and its components and related cardiometabolic risk factors in African men and women. This study used a UPLC-MS method for measuring glucocorticoid concentrations which is preferred due to its high specificity and sensitivity. In addition, all known confounders including sampling time, age, sex, relevant medication, smoking, alcohol, and adiposity, were adjusted for in the statistical analyses.

In conclusion, this study shows that circulating glucocorticoid concentrations are associated with the metabolic syndrome and related cardiometabolic variables in African men and women, but that these associations were glucocorticoid- and sex-specific. Future studies should investigate the mechanisms involved, and ensure that both cortisol and corticosterone, and sex interactions are explored.

\section{Supplementary materials}

This is linked to the online version of the paper at https://doi.org/10.1530/ EC-21-0195.

\section{Declaration of interest}

The authors declare that there is no conflict of interest that could be perceived as prejudicing the impartiality of the research reported.

\section{Funding}

The study was funded by the Academy of Medical Sciences Newton Advanced Fellowship and the South African National Research Foundation (NRF). We also acknowledge the SAMRC, with funds received from the South African National Department of Health, the UKMRC, the Newton Fund, GSK and NRF. A A C and B R W were supported by grants from British Heart Foundation and Wellcome Trust. Opinions expressed and conclusions arrived at are those of the author and are not necessarily attributed to the research funders.

\section{Author contribution statement}

$S N D, J H G, Z L, A A C$, and $B R$ W were responsible for the study conception and planning. $\mathrm{L} \mathrm{KM}, \mathrm{N} J \mathrm{C}$, and $\mathrm{S} A \mathrm{~N}$ were involved in sample and data collection. S N D and T S were responsible for measuring glucocorticoids. $\mathrm{S} N \mathrm{D}, J \mathrm{HG}$, and $\mathrm{Z} \mathrm{L}$ were involved in the data analyses. All authors were involved in the interpretation of the results and the writing of the manuscript.

\section{Acknowledgements}

The authors acknowledge participants that volunteered for this study and the staff and students of the SAMRC/Wits DPHRU and the National Health Laboratory Services for their technical support. 


\section{References}

1 Alberti KGMM, Eckel RH, Grundy SM, Zimmet PZ, Cleeman JI, Donato KA, Fruchart JC, James WPT, Loria CM, Smith SC, et al. Harmonizing the metabolic syndrome: a joint interim statement of the International Diabetes Federation Task Force on Epidemiology and Prevention; National Heart, Lung, and Blood Institute; American Heart Association; World Heart Federation; International Atherosclerosis Society; and International Association for the Study of Obesity. Circulation 2009120 1640-1645. (https://doi.org/10.1161/ CIRCULATIONAHA.109.192644)

2 Lacroix A, Feelders RA, Stratakis CA \& Nieman LK. Cushing's syndrome. Lancet 2015386 913-927. (https://doi.org/10.1016/S01406736(14)61375-1)

3 Reynolds RM, Syddall HE, Walker BR, Wood PJ \& Phillips DIW. Predicting cardiovascular risk factors from plasma cortisol measured during oral glucose tolerance tests. Metabolism: Clinical and Experimental 200352 524-527. (https://doi.org/10.1053/meta.2003.50090)

4 Ortiz R, Kluwe B, Odei JB, Tcheugui JB, Sims M, Kalyani RR, Bertoni AG, Golden SH \& Joseph JJ. The association of morning serum cortisol with glucose metabolism and diabetes: the Jackson Heart Study. Psychoneuroendocrinology 2019103 25-32. (https://doi. org/10.1016/j.psyneuen.2018.12.237)

5 Phillips DIW, Barker DJP, Fall CHD, Seckl JR, Whorwood CB, Wood PJ $\&$ Walker BR. Elevated plasma cortisol concentrations: a link between low birth weight and the insulin resistance syndrome? Journal of Clinical Endocrinology and Metabolism 199883 757-760. (https://doi. org/10.1210/jcem.83.3.4634)

6 Vogelzangs N, Beekman ATF, Dik MG, Bremmer MA, Comijs HC, Hoogendijk WJG, Deeg DJH \& Penninx BWJH. Late-life depression, cortisol, and the metabolic syndrome. American Journal of Geriatric Psychiatry 200917 716-721. (https://doi.org/10.1097/ JGP.0b013e3181aad5d7)

7 Malan L, Schutte CE, Alkerwi A, Stranges S \& Malan NT. Hypothalamic-pituitary-adrenal-axis dysregulation and double product increases potentiate ischemic heart disease risk in a black male cohort: the SABPA study. Hypertension Research 2017 40 590-597. (https://doi.org/10.1038/hr.2017.5)

8 Hüther KJ \& Scholz HR. Plasma concentrations and rates of plasma clearance and production of cortisol and corticosterone in healthy persons and in subjects with asymptomatic and clinical diabetes mellitus. Hormone and Metabolic Research 19702 357-363. (https://doi. org/10.1055/s-0028-1095054)

9 Morris DJ. Why do humans have two glucocorticoids: a question of intestinal fortitude. Steroids 2015102 32-38. (https://doi. org/10.1016/j.steroids.2015.06.017)

10 Nixon M, Mackenzie SD, Taylor AI, Homer NZM, Livingstone DE, Mouras R, Morgan RA, Mole DJ, Stimson RH, Reynolds RM, et al. ABCC1 confers tissue-specific sensitivity to cortisol versus corticosterone: a rationale for safer glucocorticoid replacement therapy. Science Translational Medicine 20168 352ra109. (https://doi. org/10.1126/scitranslmed.aaf9074)

11 Goedecke JH \& Olsson T. Pathogenesis of type 2 diabetes risk in black Africans: a South African perspective. Journal of Internal Medicine 2020 288 284-294. (https://doi.org/10.1111/joim.13083)

12 Goff LM, Ladwa M, Hakim O \& Bello O. Ethnic distinctions in the pathophysiology of type 2 diabetes: a focus on black AfricanCaribbean populations. Proceedings of the Nutrition Society 202079 184-193. (https://doi.org/10.1017/S0029665119001034)

13 Goedecke JH, Levitt NS, Lambert EV, Utzschneider KM, Faulenbach MV, Dave JA, West S, Victor H, Evans J, Olsson T, et al. Differential effects of abdominal adipose tissue distribution on insulin sensitivity in black and white South African women. Obesity 200917 1506-1512. (https://doi.org/10.1038/oby.2009.73)

14 Ntuk UE, Gill JM, Mackay DF, Sattar N \& Pell JP. Ethnic specific obesity cut-offs for diabetes risk: cross-sectional study of 489,690 UK Biobank participants. International Journal of Epidemiology 201544 i78. (https:// doi.org/10.2337/dc13-2966)

15 Lovejoy JC, De La Bretonne JA, Klemperer M \& Tulley R. Abdominal fat distribution and metabolic risk factors: effects of race. Metabolism: Clinical and Experimental 199645 1119-1124. (https://doi.org/10.1016/ s0026-0495(96)90011-6)

16 Goedecke JH, Chorell E, Livingstone DE, Stimson RH, Hayes P, Adams K, Dave JA, Victor H, Levitt NS, Kahn SE, et al. Glucocorticoid receptor gene expression in adipose tissue and associated metabolic risk in black and white South African women. International Journal of Obesity 201539 303-311. (https://doi.org/10.1038/ijo.2014.94)

17 Punyadeera C, Van Der Merwe MT, Crowther NJ, Toman M, Schlaphoff GP \& Gray IP. Ethnic differences in lipid metabolism in two groups of obese South African women. Journal of Lipid Research 2001 42 760-767. (https://doi.org/10.1016/S0022-2275(20)31638-2)

18 Schutte AE \& Olckers A. Metabolic syndrome risk in black South African women compared to Caucasian women. Hormone and Metabolic Research 200739 651-657. (https://doi.org/10.1055/s-2007-985394)

19 Malan NT, Stalder T, Schlaich MP, Lambert GW, Hamer M, Schutte AE, Huisman HW, Schutte R, Smith W, Mels CM, et al. Chronic distress and acute vascular stress responses associated with ambulatory blood pressure in low-testosterone African men: the SABPA Study. Journal of Human Hypertension 201428 393-398. (https://doi.org/10.1038/ jhh.2013.124)

20 Tolmay CM, Malan L \& Van Rooyen JM. The relationship between cortisol, C-reactive protein and hypertension in African and Causcasian women: the POWIRS study. Cardiovascular Journal of Africa 201223 78-84. (https://doi.org/10.5830/CVJA-2011-035)

21 Schutte CE, Malan L, Scheepers JD, Oosthuizen W, Cockeran M \& Malan NT. Cortisol: brain-derived neurotrophic factor ratio associated with silent ischaemia in a black male cohort: the SABPA study. Cardiovascular Journal of Africa 201627 387-391. (https://doi. org/10.5830/CVJA-2016-065)

22 Ramsay M, Crowther N, Tambo E, Agongo G, Baloyi V, Dikotope S, Gómez-Olivé X, Jaff N, Sorgho H, Wagner R, et al. H3Africa AWIGen Collaborative Centre: a resource to study the interplay between genomic and environmental risk factors for cardiometabolic diseases in four sub-Saharan African countries. Global Health, Epidemiology and Genomics 20161 e20. (https://doi.org/10.1017/gheg.2016.17)

23 Richter L, Norris S, Pettifor J, Yach D \& Cameron N. Cohort profile: Mandela's children: the 1990 birth to twenty study in South Africa. International Journal of Epidemiology 200736 504-511. (https://doi. org/10.1093/ije/dym016)

24 Ali SA, Soo C, Agongo G, Alberts M, Amenga-Etego L, Boua RP, Choudhury A, Crowther NJ, Depuur C, Gómez-Olivé FX, et al. Genomic and environmental risk factors for cardiometabolic diseases in Africa: methods used for phase 1 of the AWI-Gen population crosssectional study. Global Health Action 201811 1507133. (https://doi.org/ 10.1080/16549716.2018.1507133)

25 Friedewald WT, Levy RI \& Fredrickson DS. Estimation of the concentration of low-density lipoprotein cholesterol in plasma, without use of the preparative ultracentrifuge. Clinical Chemistry 1972 18 499-502. (https://doi.org/10.1093/clinchem/18.6.499)

26 Rstudio Team. RStudio: Integrated Development for R. PBC Boston MA: Rstudio Team, 2020. (available at: https://www.rstudio.com/)

27 Rask E, Olsson T, Söderberg S, Andrew R, Livingstone DEW, Johnson O $\&$ Walker BR. Tissue-specific dysregulation of cortisol metabolism in human obesity. Journal of Clinical Endocrinology and Metabolism 2001 86 1418-1421. (https://doi.org/10.1210/jcem.86.3.7453)

28 Gradidge PJ \& Crowther NJ. Review: Metabolic syndrome in black South African women. Ethnicity and Disease 201727 189-200. (https:// doi.org/10.18865/ed.27.2.189)

29 Weigensberg MJ, Toledo-Corral CM \& Goran MI. Association between the metabolic syndrome and serum cortisol in overweight Latino youth. Journal of Clinical Endocrinology and Metabolism 200893 1372-1378. (https://doi.org/10.1210/jc.2007-2309)

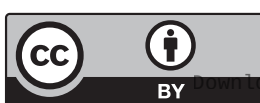

This work is licensed under a Creative Commons Attribution 4.0 International License. 
30 Guzzetti C, Pilia S, Ibba A \& Loche S. Correlation between cortisol and components of the metabolic syndrome in obese children and adolescents. Journal of Endocrinological Investigation 201437 51-56. (https://doi.org/10.1007/s40618-013-0014-0)

31 Park SB, Blumenthal JA, Lee SY \& Georgiades A. Association of cortisol and the metabolic syndrome in Korean men and women. Journal of Korean Medical Science 201126 914-918. (https://doi.org/10.3346/ jkms.2011.26.7.914)

32 Baum M \& Moe OW. Glucocorticoid-mediated hypertension: does the vascular smooth muscle hold all the answers? Journal of the American Society of Nephrology 200819 1251-1253. (https://doi.org/10.1681/ ASN.2008040410)

33 De Matteo R \& May CN. Glucocorticoid-induced renal vasodilatation is mediated by a direct renal action involving nitric oxide. American Journal of Physiology 1997273 R1972-R1979. (https://doi.org/10.1152/ ajpregu.1997.273.6.R1972)

34 Smets P, Meyer E, Maddens B \& Daminet S. Cushing's syndrome, glucocorticoids and the kidney. General and Comparative Endocrinology 2010169 1-10. (https://doi.org/10.1016/j.ygcen.2010.07.004)

35 Giannopoulos G \& Keichline D. Species-related differences in steroidbinding specificity of glucocorticoid receptors in lung. Endocrinology 1981108 1414-1419. (https://doi.org/10.1210/endo-108-4-1414)

36 Inglis GC, Ingram MC, Holloway CD, Swan L, Birnie D, Hillis WS, Davies E, Fraser R \& Connell JMC. Familial pattern of corticosteroids and their metabolism in adult human subjects - the Scottish adult twin study. Journal of Clinical Endocrinology and Metabolism 199984 4132-4137. (https://doi.org/10.1210/jcem.84.11.6146)

37 Dean M, Hamon Y \& Chimini G. The human ATP-binding cassette (ABC) transporter superfamily. Journal of Lipid Research 200142 1007-1017. (https://doi.org/10.1101/gr.184901)

38 Karssen AM, Meijer OC, Van Der Sandt ICJ, Lucassen PJ, de Lange EC, de Boer AG \& de Kloet ER. Multidrug resistance P-glycoprotein hampers the access of cortisol but not of corticosterone to mouse and human brain. Endocrinology 2001142 2686-2694. (https://doi. org/10.1210/endo.142.6.8213)

39 Raubenheimer PJ, Young EA, Andrew R \& Seckl JR. The role of corticosterone in human hypothalamic-pituitary-adrenal axis feedback. Clinical Endocrinology 200665 22-26. (https://doi. org/10.1111/j.1365-2265.2006.02540.x)

40 Walker BR, Soderberg S, Lindahl B \& Olsson T. Independent effects of obesity and cortisol in predicting cardiovascular risk factors in men and women. Journal of Internal Medicine 2000247 198-204. (https:// doi.org/10.1046/j.1365-2796.2000.00609.x)

41 Boscaro M, Giacchetti G \& Ronconi V. Visceral adipose tissue: emerging role of gluco- and mineralocorticoid hormones in the setting of cardiometabolic alterations. Annals of the New York Academy of Sciences 2012 1264 87-102. (https://doi.org/10.1111/j.17496632.2012.06597.x)
42 Varma VK, Rushing JT \& Ettinger WH. High density lipoprotein cholesterol is associated with serum cortisol in older people. Journal of the American Geriatrics Society 199543 1345-1349. (https://doi. org/10.1111/j.1532-5415.1995.tb06612.x)

43 Nanjee MN \& Miller NE. Plasma lipoproteins and adrenocortical hormones in men - positive association of low density lipoprotein cholesterol with plasma cortisol concentration. Clinica Chimica Acta: International Journal of Clinical Chemistry 1989180 113-120. (https:// doi.org/10.1016/0009-8981(89)90342-2)

44 Duma D, Collins JB, Chou JW \& Cidlowski JA. Sexually dimorphic actions of glucocorticoids provide a link to inflammatory diseases with gender differences in prevalence. Science Signaling 20103 ra74. (https://doi.org/10.1126/scisignal.2001077)

45 Quinn M, Ramamoorthy S \& Cidlowski JA. Sexually dimorphic actions of glucocorticoids: beyond chromosomes and sex hormones. Annals of the New York Academy of Sciences 20141317 1-6. (https://doi. org/10.1111/nyas.12425)

46 Kaikaew K, Steenbergen J, Van Dijk TH, Grefhorst A \& Visser JA. Sex difference in corticosterone-induced insulin resistance in mice. Endocrinology 2019160 2367-2387. (https://doi.org/10.1210/en.201900194)

47 Kuo T, McQueen A, Chen TC \& Wang JC. Regulation of glucose homeostasis by glucocorticoids. Advances in Experimental Medicine and Biology 2015 872 99-126. (https://doi.org/10.1007/978-1-4939-2895-8_5)

48 Kroon J, Pereira AM \& Meijer OC. Glucocorticoid sexual dimorphism in metabolism: dissecting the role of sex hormones. Trends in Endocrinology and Metabolism 202031 357-367. (https://doi. org/10.1016/j.tem.2020.01.010)

49 Nenke MA, Zeng A, Meyer EJ, Lewis JG, Rankin W, Johnston J, Kireta S, Jesudason S \& Torpy DJ. Differential effects of estrogen on corticosteroid-binding globulin forms suggests reduced cleavage in pregnancy. Journal of the Endocrine Society 2017 1202-210. (https://doi. org/10.1210/js.2016-1094)

50 Rebuffé-Scrive M, Walsh UA, McEwen B \& Rodin J. Effect of chronic stress and exogenous glucocorticoids on regional fat distribution and metabolism. Physiology and Behavior 199252 583-590. (https://doi. org/10.1016/0031-9384(92)90351-2)

51 Eisenhofer G, Peitzsch M, Kaden D, Langton K, Pamporaki C, Masjkur J, Tsatsaronis G, Mangelis A, Williams TA, Reincke M, et al. Reference intervals for plasma concentrations of adrenal steroids measured by LC-MS/MS: impact of gender, age, oral contraceptives, body mass index and blood pressure status. Clinica Chimica Acta: International Journal of Clinical Chemistry 2017470 115-124. (https:// doi.org/10.1016/j.cca.2017.05.002)

52 Motala AA, Esterhuizen T, Gouws E, Pirie FJ \& Omar MA. Diabetes and other disorders of glycemia in a rural South African community: prevalence and associated risk factors. Diabetes Care 200831 1783-1788. (https://doi.org/10.2337/dc08-0212)

Received in final form 28 June 2021

Accepted 14 July 2021

Accepted Manuscript published online 14 July 2021 https://ec.bioscientifica.com https://doi.org/10.1530/EC-21-0195
(C) 2021 The authors Published by Bioscientifica Ltd

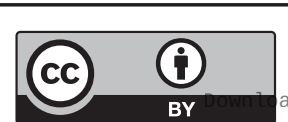

This work is licensed under a Creative Commons Attribution 4.0 International License. ded from Bioscientifica com at 04/26/2023 11:21:03AM 